\title{
Charged Higgs pair production and neutrino effects on the triple Higgs coupling
}

\author{
Julien Baglio* \\ Institut für Theoretische Physik, Eberhard Karls Universität Tübingen, \\ Auf der Morgenstelle 14, 72076 Tübingen (Germany) \\ E-mail: julien.baglio@tuebingen.de
}

Higgs pair production is one of the primary goals of the LHC program. Investigating the effects beyond the Standard Model (BSM) is then of high interest. Two cases are presented to exemplify the impact of BSM physics on Higgs pair production and on the triple Higgs coupling: first a review on charged Higgs pair production mostly in the context of Two-Higgs-Doublet of type II and in particular the Minimal Supersymmetric SM, second a study of the one-loop effects of a heavy neutrino on the triple Higgs coupling.

Prospects for Charged Higgs Discovery at Colliders 3-6 October 2016

Uppsala, Sweden

${ }^{*}$ Speaker. 


\section{Introduction}

Since the ATLAS and CMS collaborations at the CERN Large Hadron Collider (LHC) discovered in 2012 a particle which properties are compatible with the Standard Model (SM) Higgs boson hypothesis $[1,2]$, the detailed study of its properties have started. Run I results at 7-8 $\mathrm{TeV}$ do not display any deviations with respect to the SM [3-6], as well as the first results of the Run II at $13 \mathrm{TeV}$ [7-9]. The measure of the triple Higgs coupling would allow for a direct probe of the scalar potential that is directly responsible for the electroweak symmetry breaking (EWSB) [10-14]. This is one of the major goals of the LHC and of the future planned colliders such as the electron-positron International Linear Collider (ILC) or the Future Circular Collider in hadron mode (FCC-hh), a potential $100 \mathrm{TeV}$ follow-up of the LHC.

In the past few years there has been numerous studies on the production of Higgs boson pairs, that is the main production mechanism that allows for the measure of the triple Higgs coupling, see reviews in Refs. [15 - 17] for the latest theoretical developments in the SM. Beyond-the-SM (BSM) effects on Higgs pair production have been studied in many different frameworks and in particular in the Two-Higgs-Doublet-Model (2HDM) (see e.g. Refs. [18-20] and the results collected in Ref. [17]) and in the minimal supersymmetric extension of the SM (MSSM) which is a particular type of $2 \mathrm{HDM}$ in the Higgs sector (see for example Ref. [21]). In 2HDMs there are five Higgs bosons in the spectrum, and in particular there are two charged Higgs bosons $H^{ \pm}$, for a review see Ref. [22]. To allow for the measure of the triple Higgs coupling involving charged Higgs bosons it is necessary to produce pairs of charged Higgs bosons.

In the following we will give a brief overview of the theoretical predictions for charged Higgs boson pair production at the LHC. As an other example of BSM effects on the triple Higgs coupling we will then present in a second part a recent study [23] on the impact of an heavy neutrino on the one-loop corrected triple Higgs coupling in a simplified model. We stress that the results in the latter case are quite generic and could be applicable in any model containing heavy fermions that couple via the neutrino portal.

\section{Charged Higgs boson pair production at the LHC}

The Higgs sector of a 2HDM contains two Higgs doublets leading to 5 Higgs bosons in the spectrum, two of which are the charged Higgs bosons $H^{ \pm}$. In a type I 2HDM only one of the Higgs doublets couples to all fermions while in a type II 2HDM one doublet couples to up-type quarks and the second doublet couples to down-type quarks and charged leptons. In the MSSM, the Higgs sector is a type II $2 \mathrm{HDM}$ and can be parameterised by two parameters, the ratio of the two vacuum expectation values $\tan \beta=v_{2} / v_{1}$ and the mass of the pseudo-scalar Higgs boson $M_{A}$. More parameters are required in a general $2 \mathrm{HDM}$. For a recent review on the prospects for charged Higgs bosons searches at the LHC see Ref. [24].

The constraints on the charged Higgs bosons come from precision measurements as well as from direct searches at LEP, the Tevatron and the LHC. The former are the most stringent limits on the charged Higgs boson mass, especially in a type II 2HDM. The global fit [25] including all flavour observables was updated in 2015 especially for the $B \rightarrow X_{s} \gamma$ observable and gives a lower limit of $M_{H^{ \pm}} \geq 493 \mathrm{GeV}$ [26], when including higher-order corrections in $\operatorname{BR}\left(B \rightarrow X_{s} \gamma\right)$ 

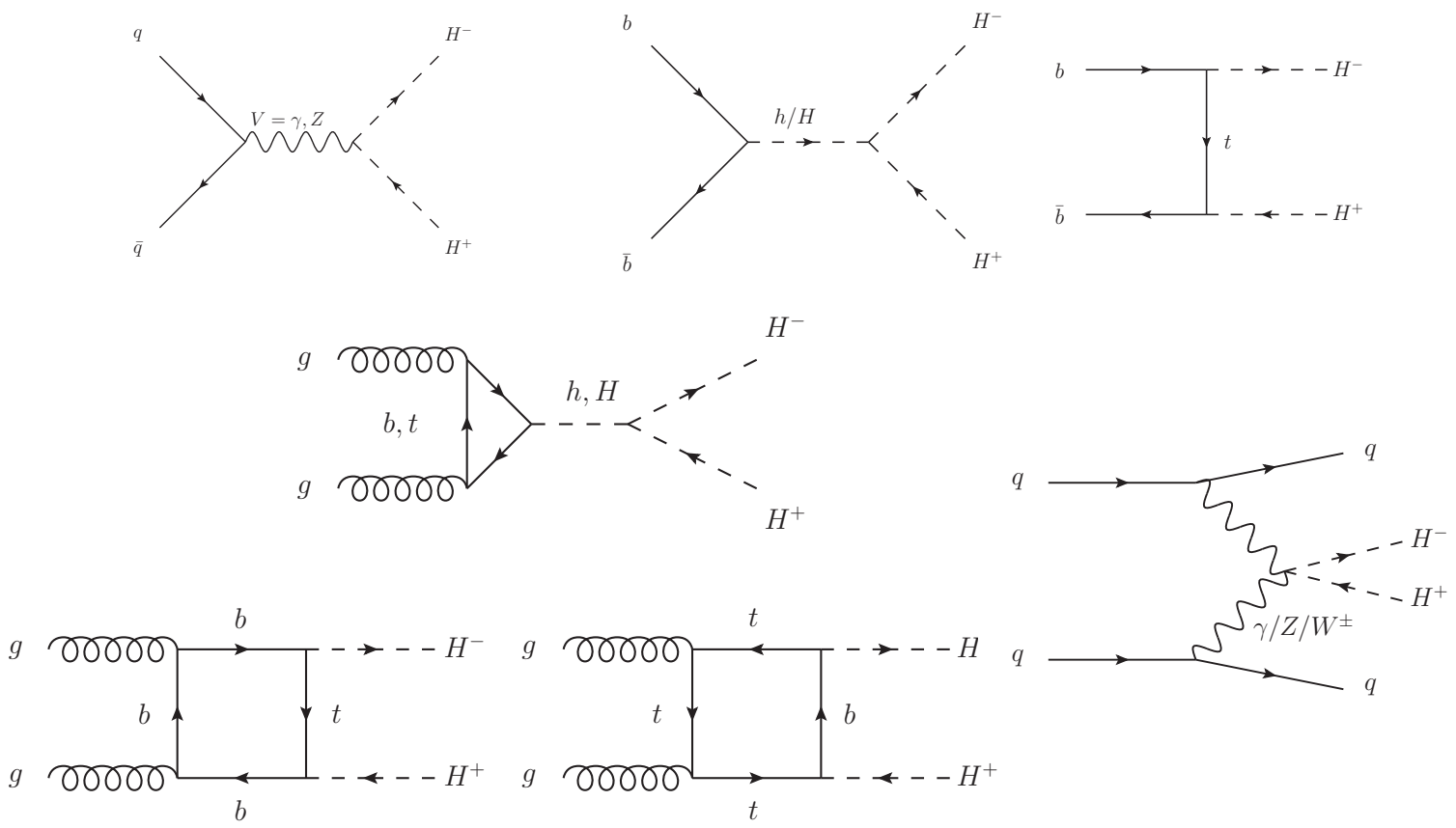

Figure 1: Generic LO Feynman diagrams for the main production channels of charged Higgs boson pair production: Drell-Yan (upper left), bottom quark fusion (upper right), gluon fusion (lower left) and VBF (lower right).

prediction [27]. A type I 2HDM escapes this flavour limit and the limits are much weaker, mainly coming from direct searches at LEP, with $M_{H^{ \pm}} \gtrsim 72.5 \mathrm{GeV}$, while the limit for a type II 2HDM is $M_{H^{ \pm}} \gtrsim 80 \mathrm{GeV}$ [28]. The Tevatron searches [29] are now superseeded by the LHC results. For example LHC Run I data excludes charged Higgs boson masses of $M_{H^{ \pm}}<140 \mathrm{GeV}$ in the MSSM with an $m_{h}^{\text {mod- }}$ scenario [30, 31]. The first Run II results at $13 \mathrm{TeV}$ have improved the direct limits in the high-mass range, see Refs. [32-34].

There are three main classes of charged Higgs boson pair production at the LHC. The dominant channel at low $\tan \beta \lesssim 30$ is Drell-Yan production, including the bottom-quark initiated channel $b \bar{b} \rightarrow H^{+} H^{-}$, then gluon fusion which is dominant at high $\tan \beta \gtrsim 50$ and finally vector boson fusion (VBF) which is usually the second production channel. Generic leading order (LO) Feynman diagrams are depicted in Fig. 1. The cross section are generically small as exemplified in Fig. 2 for Drell-Yan production (including bottom-quark fusion) and gluon fusion at next-to-leading order (NLO) in QCD, at different values of $\tan \beta$. This means that high luminosity is required to observe the possible production of a pair of charged Higgs bosons, except in the case of resonant production, that could lead to a sizeable enhancement, see below for a discussion of this possibility especially in a type I $2 \mathrm{HDM}$.

\subsection{Drell-Yan and $b \bar{b}$ production}

The Drell-Yan process $q \bar{q} \rightarrow H^{+} H^{-}$provides the largest production channel at low $\tan \beta \lesssim 30$. The LO cross section was calculated in the 1980s [36-38], then reproduced and studied later in the 2000s $[39,40]$. The NLO QCD corrections induce a $+27 \%(+17 \%)$ increase at $14 \mathrm{TeV}$ for 

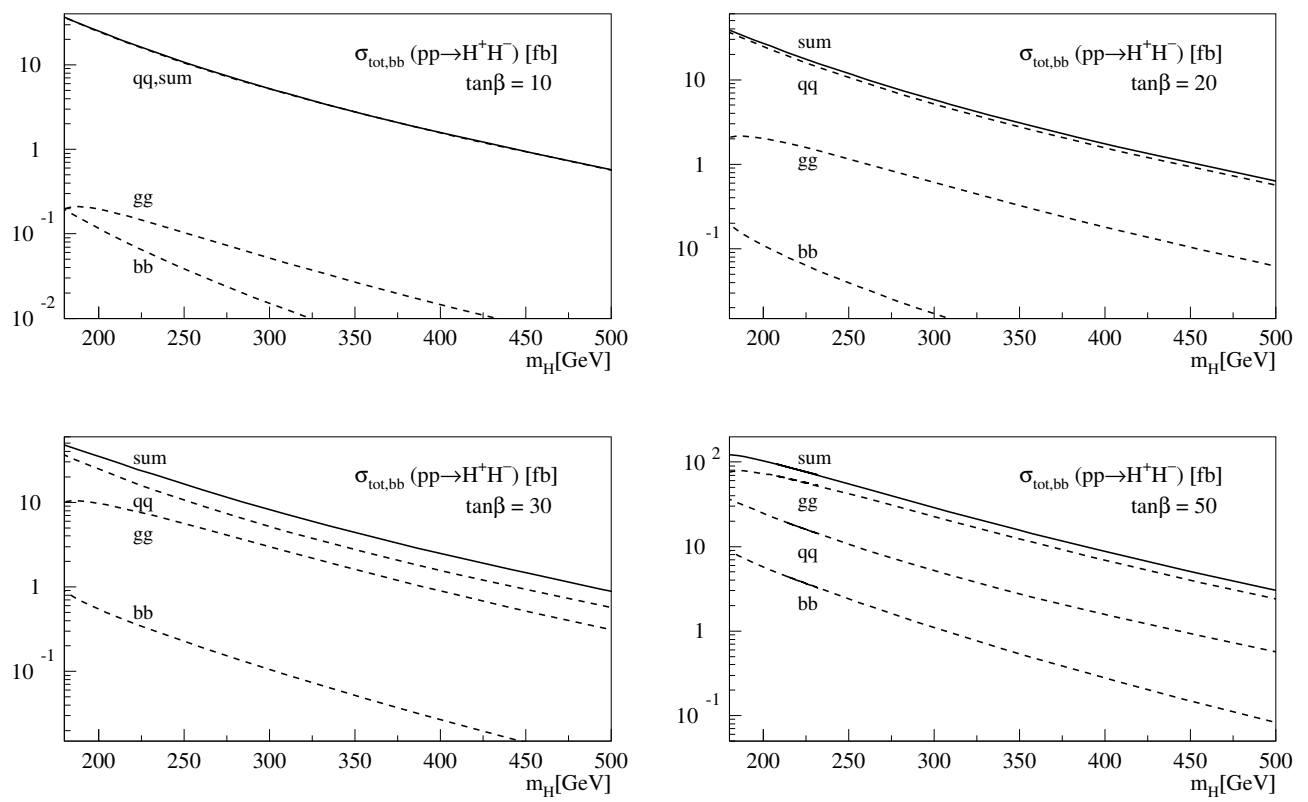

Figure 2: NLO QCD production cross sections (in fb) of $p p \rightarrow H^{+} H^{-}$in the Drell-Yan channel (labelled as $q q$ ), the bottom-quark fusion channel (labelled as $b b$ ) and in the gluon fusion channel (labelled as $g g$ ) as a function of the charged Higgs boson mass $m_{H}$ (in $\mathrm{GeV}$ ). Four different values of $\tan \beta$ are investigated. Taken from Ref. [35].

$M_{H^{ \pm}}=160(500) \mathrm{GeV}$, with $\sigma=58(0.23) \mathrm{fb}[35,41]$. The cross section has no dependence on $\tan \beta$. The scale uncertainty is of order $\pm 25 \%$. The supersymmetric (SUSY) QCD corrections have been calculated in Ref. [41] and are negligible.

The study of the additional bottom-quark fusion subprocess started in the 2000s. The $b \bar{b}$ process suffers from an ambiguity: $b$-quarks in the proton come from a gluon splitting and the process could be viewed either as a direct bottom-quark fusion production [39, 42] or as $H^{+} H^{-}$ production in association with $b$-jets, $g g \rightarrow H^{+} H^{-} b \bar{b}$ [42, 43]. It was found at first that direct production was one order of magnitude larger. This was related to the key issue of defining the right factorisation scale $\mu_{F}$ as $\log \left(\mu_{F} / m_{b}\right)$ terms that are resummed in the $b$-parton picture could overestimate the cross section if $\mu_{F}$ were too big.

The calculation of the NLO QCD corrections [35, 44] solved the issue and confirmed that the correct choice is $\mu_{F}=\frac{1}{2} M_{H^{ \pm}}$. The QCD corrections add $\sim+55 \%$ to the total cross section in the $2 \mathrm{HDM}$ and the scale uncertainty is of order $\pm 25 \%$ [35]. The cross section is dependent on $\tan \beta$. In the MSSM the SUSY-QCD corrections are dominated by negative resummed $\Delta_{b}$-terms in the bottom quark Yukawa coupling and depend strongly on the MSSM spectrum [35, 44].

\subsection{The vector boson fusion channel}

The VBF channel is usually the second most important production channel at the LHC. The first approximate calculation was done in the 1980s for the $40 \mathrm{TeV}$ Superconducting Super Collider [45]. The LO full calculation in the MSSM for the LHC was completed in 2001 for the total cross section as well as for the differential distributions [42], but has to be taken with care as it 
uses an explicit quark mass in the $\mathrm{MeV}$ range to regularize the collinear singularities due to photon exchange, that is not consistent with the parton picture in QCD. The cross section displays no dependence on $\tan \beta$ as exemplified in Fig. 3 (left).

The LO cross section was recalculated for a general 2HDM (of any type) and analysed in Ref. [40]. It was shown that resonant effects in the $s$-channel due to heavy Higgs bosons are possible and could enhance the cross section up to the picobarn level, see Fig. 3 (right). The NLO QCD corrections are still unknown.
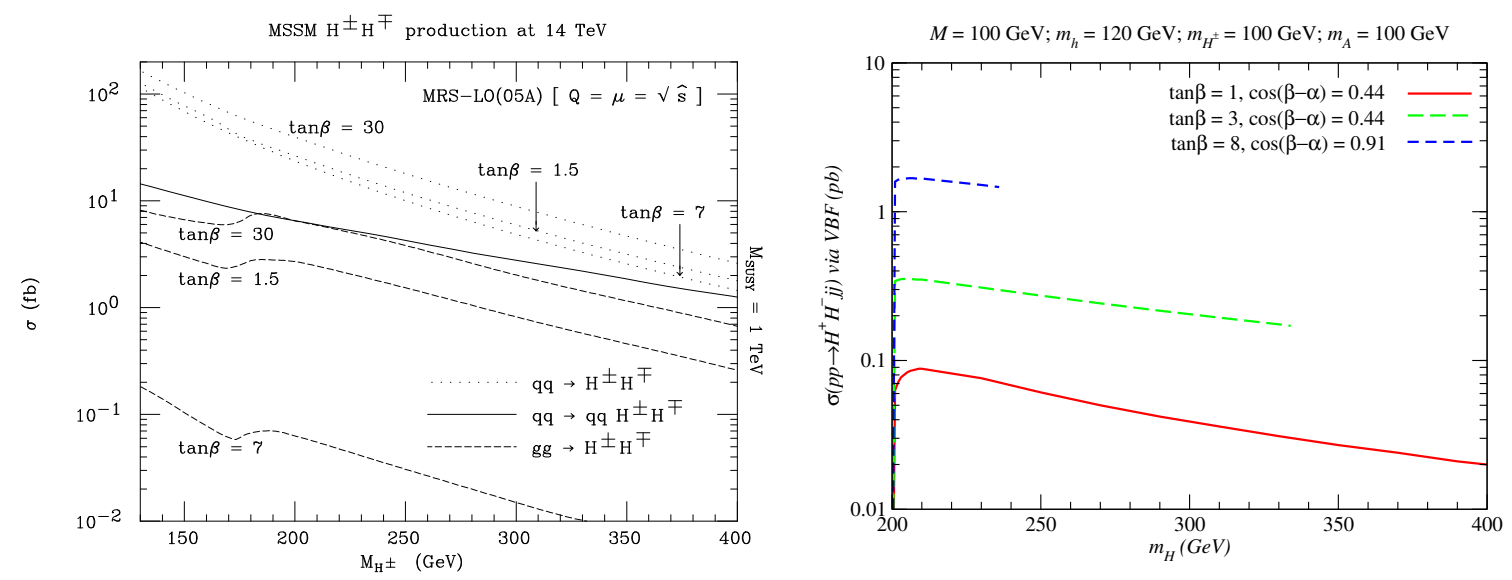

Figure 3: Left: The Drell-Yan, VBF and gluon fusion $H^{+} H^{-}$production cross section at the $14 \mathrm{TeV}$ LHC (in $\mathrm{fb}$ ) as a function of the charged Higgs boson mass $M_{H^{ \pm}}$(in GeV) in the MSSM with a SUSY scale of 1 $\mathrm{TeV}$, for different values of $\tan \beta$. Taken from Ref. [42]. Right: The VBF $H^{+} H^{-}$production cross section (in pb) as a function of the heavy CP-even Higgs boson mass $m_{H}$ (in $\mathrm{GeV}$ ) in a $2 \mathrm{HDM}$ for different values of $\tan \beta$ and fixed values for $m_{h}, m_{H^{ \pm}}$and $m_{A}$, complying with all known constraints on the model. Taken from Ref. [40].

\subsection{The gluon fusion channel}

We finish this review of the main production channels with the one-loop initiated gluon fusion production that is known at LO. This is the dominant channel for $\tan \beta \gtrsim 50$ and could feature resonant effects. The earliest calculations can be traced back to the 1980s, with 2HDM approximate calculations including only the triangle quark loop [46]. This was followed by the exact calculation of the triangle quark loop, accompanied by the box loop in the heavy quark limit in the 2HDM of type I and II [47]. The calculation in the MSSM with loops of heavy squarks was completed at the sime time [48].

The process was studied again at the end of the 1990s and after the completion of the full exact quark contributions $[49,50]$ (the first calculation being wrong and corrected by the second calculation) the full calculation in the MSSM and in 2HDMs was completed by different groups at nearly the same time [39, 51, 52] and later including CP violating effects [53]. The matching with parton shower has been available since 2015 [54].

The squark loops lead to a large enhancement of the total cross section [52] as exemplified in Fig. 4 (left). Depending on the MSSM parameters, the cross section can reach $200 \mathrm{fb}$ at high $\tan \beta$. The scale uncertainty is estimated to be of order $\pm 50 \%$ [35]. In a 2HDM (in particular of type I) resonant effects are possible and lead to pb cross sections as studied in Ref. [40] and exemplified in Fig. 4 (right). This makes this channel a very good probe of the $h H^{ \pm} H^{-}$and $H H^{ \pm} H^{-}$couplings. 

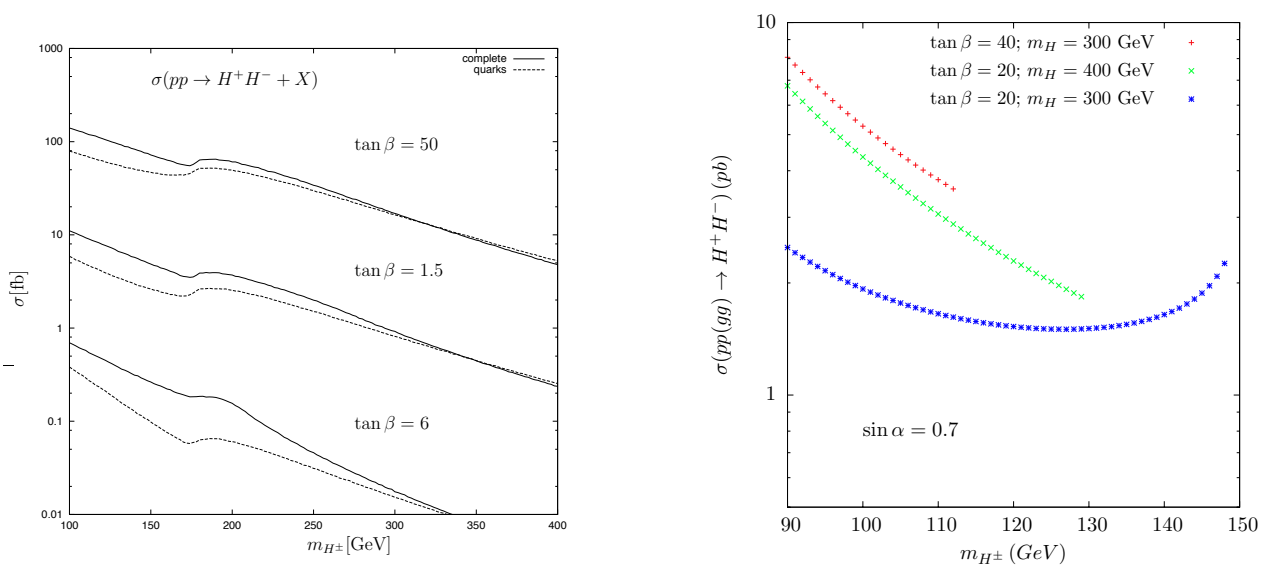

Figure 4: Left: The gluon fusion production cross section $p p \rightarrow H^{+} H^{-}$at the $14 \mathrm{TeV}$ LHC as a function of the charged Higgs boson mass $m_{H^{ \pm}}$(in GeV). Left: Cross sections (in fb) in the MSSM for different $\tan \beta$ values, with the quark contributions only (in dashed) and with the full contributions including squark loops (in solid). Taken from Ref. [52]. Right: Cross sections (in pb) in a 2HDM with resonant effects for different values of $\tan \beta$ and of the heavy CP-even Higgs boson mass $m_{H}$, complying with all known constraints on the model. Taken from Ref. [40].

\section{Heavy neutrino impact on the triple Higgs coupling}

Since the Super-Kamiokande experiment in 1998 it is now experimentally established that the neutrino flavours oscillate [55]. This calls for a BSM explanation as it implies that neutrinos are not massless. One of the simplest possibilities to explain neutrino masses is to add new fermionic heavy gauge singlets that play the role of right-handed neutrinos in a seesaw mechanism, see Ref. [56] for a review on low-scale seesaw phenomenology at colliders.

We present a study of the impact of these new heavy neutrinos on the triple Higgs coupling by considering a simplified $3+1$ model where the SM is minimally modified to account for 3 light massive Dirac neutrinos and one heavy sterile Dirac neutrino. We recall that the experimental prospects we take for the sensitivity to $\lambda_{H H H}$ are the following: $\sim 35 \%$ at the high-luminosity LHC when combining the $\sim 50 \%$ sensitivity taken from Ref. [57]; $\sim 10 \%$ at the ILC at $1 \mathrm{TeV}$ with 5 $\mathrm{ab}^{-1}$ [58]; and finally $\sim 5 \%$ sensitivity at the FCC-hh when combining the $\sim 8 \%$ per experiment with $3 \mathrm{ab}^{-1}$ taken from Ref. [59].

\subsection{The simplified model and its constraints}

To illustrate the impact of a new, $\mathrm{TeV}$ scale fermion we use a simplified model with 3 light active and one heavy sterile neutrinos $n_{i}$, parametrized by the masses $m_{i}$ and the active-sterile mixing matrix $B_{j k}, i, k=1 \ldots 4$ and $j=1 \ldots 3$. The Lagrangian contains the following neutrino interactions,

$$
\begin{aligned}
& \mathscr{L}_{n}=-\frac{g_{2}}{\sqrt{2}} \bar{\ell}_{i} \gamma^{\mu} W_{\mu}^{-} B_{i j} P_{L} n_{j}+\text { h.c. } \\
& -\frac{g_{2}}{2 \cos \theta_{W}} \bar{n}_{i} \gamma^{\mu} Z_{\mu}\left(B^{\dagger} B\right)_{i j} P_{L} n_{j}-\frac{g_{2}}{2 M_{W}} \bar{n}_{i} H\left(B^{\dagger} B\right)_{i j}\left(m_{n_{i}} P_{L}+m_{n_{j}} P_{R}\right) n_{j} .
\end{aligned}
$$

The active-sterile mixing matrix $B$ is build from the PMNS matrix [60, 61] extended to 4 neutrinos with no $\mathrm{CP}$ violation in the neutrino sector. 
The new heavy neutrino generates new triangle one-loop contributions for the triple Higgs coupling $\lambda_{H H H}$ and lead to modifications in the Higgs and weak bosons self-energies. Amongst the experimental constraints that are applicable to the model, electroweak precision observables (EWPO) are the strongest $[62,63]$. Constraints coming from neutrinoless $\beta$-decay do not apply and flavour violating Higgs decays are less constraining than EWPO. We are lead to the following constraints on the active-sterile mixing matrix,

$$
B_{e 4} \leq 0.041, B_{\mu 4} \leq 0.030, B_{\tau 4} \leq 0.087 .
$$

We also apply theoretical constraints on the perturbativity of the triple Higgs coupling as well as on the width of the heavy neutrino,

$$
\left(\frac{g_{2} m_{n_{4}}}{2 M_{W}} \max \left|\left(B^{\dagger} B\right)_{i 4}\right|\right)^{3}<16 \pi(2 \pi), \quad \Gamma_{n_{4}} \leq 0.06 m_{n_{4}} .
$$

$2 \pi$ stands for a tighter perturbativity bound roughly equivalent to a two-loop analysis carried in the SM.

\subsection{Numerical results}

To illustrate the impact of the heavy neutrino in our simplified model we display in Fig. 5 (left) the dependence of the full one-loop corrections (including the SM contributions) on the momentum $q_{H}^{*}$ of the off-shell Higgs splitting in two on-shell Higgs bosons $H\left(q_{H}^{*}\right) \rightarrow H H$, for different heavy neutrino mass values $m_{n_{4}}$. We assume the maximal allowed value for $B_{\tau 4}=0.087$ while $B_{e 4}=$ $B_{\mu 4}=0$. Similar plots are obtained for the other maximally allowed mixings. $m_{n_{4}}=2.7 \mathrm{TeV}$ corresponds to the choice of a neutrino Yukawa coupling equal to the top quark Yukawa coupling, $m_{n_{4}}=7 \mathrm{TeV}$ is the maximal mass allowed by the tight perturbativity bound while $m_{n_{4}}=9 \mathrm{TeV}$ is the maximal value allowed by the width bound. The largest positive one-loop correction is obtained at $q_{H}^{*} \simeq 500 \mathrm{GeV}$ and becomes smaller for a heavier neutrino (eventually going negative). At large $q_{H}^{*} \simeq 2.5 \mathrm{TeV}$ the largest negative one-loop correction is obtained and the heavier the neutrino is, the larger the correction becomes.

In Fig. 5 (right) we display the contour map of the genuine correction $\Delta^{\mathrm{BSM}}$ (in percent) due to the heavy neutrino, in the plane $\left(m_{n_{4}},\left|B_{\tau 4}\right|^{2}\right)$. The off-shell Higgs boson momentum is fixed to $q_{H}^{*}=2.5 \mathrm{TeV}$. The red line displays the tight perturbativity bound. We obtain corrections as large as $\sim+30 \%$ when taking into account the experimental and theoretical constraints, at the limit of the high-luminosity LHC and clearly visible at the ILC or at the FCC-hh. Again a similar behaviour can be obtained for the active-sterile mixings $B_{e 4}$ and $B_{\mu 4}$.

\section{Outlook}

The production of a Higgs boson pair is one of the main goals of the high-luminosity run of the LHC and of the future colliders, in order to ultimately measure the triple Higgs coupling. Assessing the effects of BSM models on the Higgs sector and in particular on the triple Higgs coupling is thus an essential task.

As an illustrative example of such studies we have presented in a first part a review of the current status for charged Higgs pair production at the LHC in the 2HDM and the MSSM, focusing 

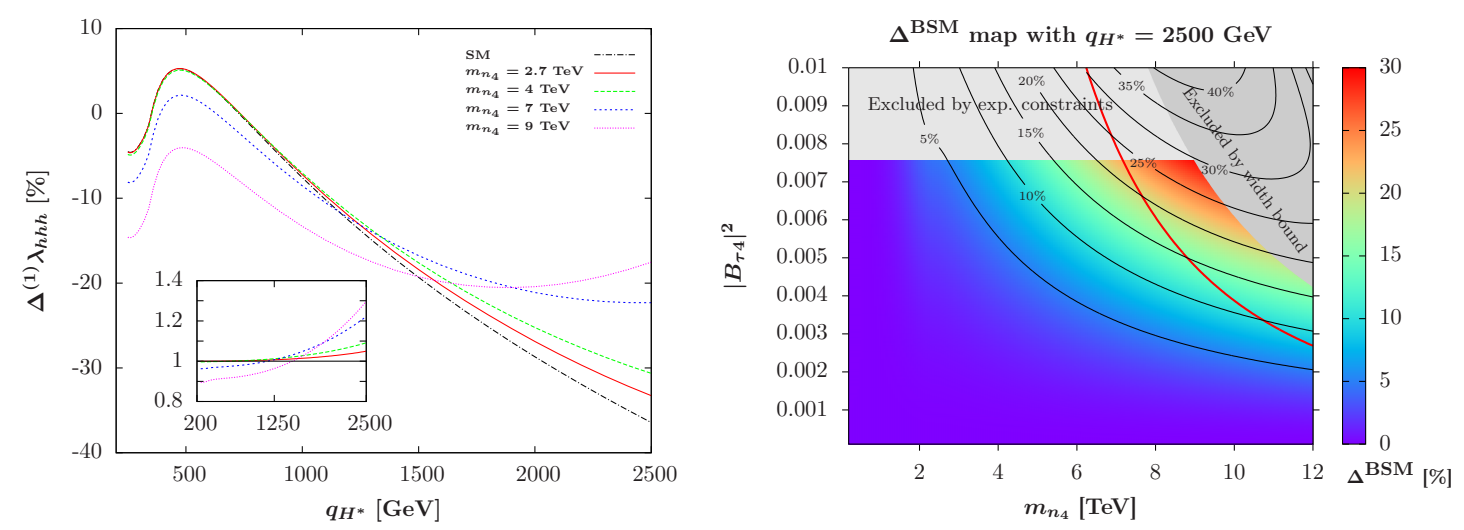

Figure 5: Left: One-loop corrections to $\lambda_{H H H}$ (in \%) as a function of the off-shell Higgs momentum $q_{H}^{*}$ (in $\mathrm{GeV}$ ) of the splitting $H\left(q_{H}^{*}\right) \rightarrow H H$, with the neutrino parameter $B_{\tau 4}=0.087$. Right: Contour map of the neutrino corrections $\Delta^{\mathrm{BSM}}$ (in \%) as a function of the two neutrino parameters $m_{n_{4}}$ (in GeV) and $\left|B_{\tau 4}\right|^{2}$ at a fixed $q_{H}^{*}=2.5 \mathrm{TeV}$. Both figures are taken from Ref. [23].

on the main production channels: Drell-Yan production including bottom-quark effects in the initial state, vector boson fusion and gluon fusion. While the dominant channel at low $\tan \beta$, Drell-Yan production, is known at NLO in (SUSY-) QCD, vector boson and gluon fusions are only known at LO. Precision may be improved in particular in the vector boson fusion channel.

We have presented in a second part the BSM effect induced by a heavy neutrino on the triple Higgs coupling. Using a simplified model with one heavy neutrino, effects as large as $+30 \%$ can be found, that are clearly measurable at future colliders. The triple Higgs coupling is thus a new observable for neutrino physics that could be used to probe neutrino mass regimes hard to access otherwise and would constraint the active-sterile mixing.

\section{Acknowledgments}

The author would like to warmly thank the organisers for the invitation and for the very nice atmosphere of this workshop. Discussions with Michael Spira are also acknowledged. He acknowledges the support from the Institutional Strategy of the University of Tübingen (DFG, ZUK 63) and from the DFG Grant JA 1954/1.

\section{References}

[1] ATLAS collaboration, G. Aad et al., Phys. Lett. B716 (2012) 1-29, [1207. 7214].

[2] CMS collaboration, S. Chatrchyan et al., Phys. Lett. B716 (2012) 30-61, [1207 . 7235].

[3] ATLAS collaboration, G. Aad et al., Eur. Phys. J. C75 (2015) 476, [1506 . 05669 ].

[4] ATLAS collaboration, G. Aad et al., Eur. Phys. J. C76 (2016) 6, [1507. 04548 ].

[5] CMS collaboration, S. Chatrchyan et al., Phys. Rev. D89 (2014) 092007, [1312 . 5353].

[6] CMS collaboration, V. Khachatryan et al., Phys. Rev. D92 (2015) 012004, [1411. 3441 ].

[7] ATLAS collaboration, ATLAS-CONF-2016-081 (2016). 
[8] CMS collaboration, CMS-PAS-HIG-16-020 (2016).

[9] CMS collaboration, CMS-PAS-HIG-16-033 (2016).

[10] P. W. Higgs, Phys. Lett. 12 (1964) 132-133.

[11] F. Englert and R. Brout, Phys. Rev. Lett. 13 (1964) 321-323.

[12] P. W. Higgs, Phys. Rev. Lett. 13 (1964) 508-509.

[13] G. S. Guralnik, C. R. Hagen and T. W. B. Kibble, Phys. Rev. Lett. 13 (1964) 585-587.

[14] P. W. Higgs, Phys. Rev. 145 (1966) 1156-1163.

[15] N. Arkani-Hamed, T. Han, M. Mangano and L.-T. Wang, Phys. Rept. 652 (2016) 1-49, [1511.06495].

[16] J. Baglio, A. Djouadi and J. Quevillon, Rept. Prog. Phys. 79 (2016) 116201, [1511. 07853 ].

[17] LHC Higgs Cross Section Working Group collaboration, D. de Florian et al., 1610.07922.

[18] J. Baglio, O. Eberhardt, U. Nierste and M. Wiebusch, Phys. Rev. D90 (2014) 015008, [1403.1264].

[19] B. Hespel, D. Lopez-Val and E. Vryonidou, JHEP 09 (2014) 124, [1 407 . 0281].

[20] H. E. Haber and O. Stål, Eur. Phys. J. C75 (2015) 491, [1507. 04281$].$

[21] J. Cao, Z. Heng, L. Shang, P. Wan and J. M. Yang, JHEP 04 (2013) 134, [1301. 6437 ].

[22] G. C. Branco, P. M. Ferreira, L. Lavoura, M. N. Rebelo, M. Sher and J. P. Silva, Phys. Rept. 516 (2012) 1-102, [1106.0034].

[23] J. Baglio and C. Weiland, Phys. Rev. D94 (2016) 013002, [1603.00879].

[24] A. G. Akeroyd et al., 1607.01320.

[25] H. Flacher, M. Goebel, J. Haller, A. Hocker, K. Monig and J. Stelzer, Eur. Phys. J. C60 (2009) 543-583, [0811.0009].

[26] T. Enomoto and R. Watanabe, JHEP 05 (2016) 002, [1 511.05066 ].

[27] M. Misiak et al., Phys. Rev. Lett. 114 (2015) 221801, [1503.01789].

[28] LEP, DELPHI, OPAL, ALEPH, L3 collaboration, G. Abbiendi et al., Eur. Phys. J. C73 (2013) 2463, [1301.6065].

[29] CDF, D0 collaboration, P. Gutierrez, PoS CHARGED2010 (2010) 004.

[30] ATLAS collaboration, G. Aad et al., JHEP 03 (2015) 088, [1412 . 6663].

[31] ATLAS collaboration, G. Aad et al., JHEP 03 (2016) 127, [1512. 0370 4].

[32] ATLAS collaboration, ATLAS-CONF-2016-088 (2016).

[33] ATLAS collaboration, ATLAS-CONF-2016-089 (2016).

[34] CMS collaboration, CMS-PAS-HIG-16-030 (2016).

[35] A. Alves and T. Plehn, Phys. Rev. D71 (2005) 115014, [hep-ph/0 503135$].$

[36] K. D. Lane, eConf C8206282 (1982) 222-241.

[37] E. Eichten, I. Hinchliffe, K. D. Lane and C. Quigg, Rev. Mod. Phys. 56 (1984) 579-707.

[38] N. G. Deshpande, X. Tata and D. A. Dicus, Phys. Rev. D29 (1984) 1527. 
[39] A. A. Barrientos Bendezú and B. A. Kniehl, Nucl. Phys. B568 (2000) 305-318, [hep-ph/9908385].

[40] M. Aoki, R. Guedes, S. Kanemura, S. Moretti, R. Santos and K. Yagyu, Phys. Rev. D84 (2011) 055028, [1104.3178].

[41] A. Djouadi and M. Spira, Phys. Rev. D62 (2000) 014004, [hep-ph/9912476].

[42] S. Moretti, J. Phys. G28 (2002) 2567-2582, [hep-ph/0102116].

[43] S. Moretti and J. Rathsman, Eur. Phys. J. C33 (2004) 41-52, [hep-ph/ 0308215$].$

[44] H. Hong-Sheng, M. Wen-Gan, Z. Ren-You, J. Yi, H. Liang and X. Li-Rong, Phys. Rev. D71 (2005) 075014, [hep-ph/0502214].

[45] O. J. P. Éboli, G. C. Marques, S. F. Novaes, A. A. Natale and J. M. R. Pulido, Phys. Rev. D37 (1988) 837.

[46] E. Eichten, I. Hinchliffe, K. D. Lane and C. Quigg, in ELECTROWEAK SYMMETRY BREAKING. PROCEEDINGS, WORKSHOP, BERKELEY, USA, JUNE 3-22, 1984, 1984.

[47] R. Foot, H. Lew and G. C. Joshi, Phys. Rev. D37 (1988) 3161.

[48] S. S. D. Willenbrock, Phys. Rev. D35 (1987) 173.

[49] Y. Jiang, L. Han, W.-G. Ma, Z.-H. Yu and M. Han, J. Phys. G23 (1997) 1151, [hep-ph/9703275].

[50] A. Krause, T. Plehn, M. Spira and P. M. Zerwas, Nucl. Phys. B519 (1998) 85-100, [hep-ph/9707430].

[51] Y. Jiang, W.-g. Ma, L. Han, M. Han and Z.-h. Yu, J. Phys. G24 (1998) 83-95, [hep-ph/9708421].

[52] O. Brein and W. Hollik, Eur. Phys. J. C13 (2000) 175-184, [hep-ph/9908529].

[53] Y. Jiang, W.-G. Ma, L. Han and H.-S. Hou, Commun. Theor. Phys. 36 (2001) 61-64, [hep-ph/0012079].

[54] V. Hirschi and O. Mattelaer, JHEP 10 (2015) 146, [1507.00020].

[55] Super-Kamiokande collaboration, Y. Fukuda et al., Phys. Rev. Lett. 81 (1998) 1562-1567, [hep-ex/9807003].

[56] F. F. Deppisch, P. S. Bhupal Dev and A. Pilaftsis, New J. Phys. 17 (2015) 075019, [1502. 06541 ].

[57] CMS collaboration, CMS-PAS-FTR-15-002 (2015).

[58] K. Fujii et al., 1506.05992.

[59] H.-J. He, J. Ren and W. Yao, Phys. Rev. D93 (2016) 015003, [1506. 03302 ].

[60] B. Pontecorvo, Sov. Phys. JETP 6 (1957) 429.

[61] Z. Maki, M. Nakagawa and S. Sakata, Prog. Theor. Phys. 28 (1962) 870-880.

[62] F. del Aguila, J. de Blas and M. Perez-Victoria, Phys. Rev. D78 (2008) 013010, [0 803.4008 ].

[63] J. de Blas, EPJ Web Conf. 60 (2013) 19008, [1307.6173]. 